\title{
ELECTRONIC STRUCTURE OF SEMICONDUCTOR SURFACES STUDIED BY PHOTOELECTRON SPECTROSCOPY
}

\author{
P.O. NILSSON \\ Department of Physics, Chalmers University of Technology \\ 41296 Göteborg, Sweden
}

\begin{abstract}
The present status of the understanding of the electronic structure of semiconductor surfaces is reviewed. In particular the interplay between photoelectron spectroscopy and calculations of the surface electronic structure is stressed.
\end{abstract}

PACS numbers: $79.60 .-\mathrm{i}$

\section{Introduction}

Surface science goes back to the beginning of the 19 th century. It was, however, only with the development of ultra high vacuum (UHV) technology in the 1960 's that reproducible experirriental results from surfaces on the atomic scale could be obtained. At about the same time high speed computers appeared which made it possible to apply realistic computations of surface properties.

The last decade has seen a large progress in surface science and the field is under rapid development. This is largely due to the continuous development of the experimental and the theoretical methods. Many basic properties of surfaces are now in principle understood. Much more research is however necessary to achieve detailed descriptions, in particular of complex systems such as interfaces, overlayers and multilayers. A co-ordinated interplay between theory and experiment is desirable to achieve an efficient progress.

In this article we will illustrate the present understanding of semiconductor surfaces as achieved mainly by photoelectron spectroscopy using synchrotron radiation. We will choose three examples, namely results from (i) core level studies on a clean surface, (ii) valence band studies of a clean surface, and (iii) core level and valence band studies on an adsorbate system. Before presenting these results we will summarise the present understanding of the atomic and the electronic structure of crystalline serriconductor surfaces. 


\section{The atomic structure of surfaces}

A working definition of a surface is that it comprises the 3-4 outermost atomic layers of a solid. The atomic positions in this region are always different from the corresponding ones in the bulk, because the forces on the atoms are modified due to the vicinity of the vacuum region. In some cases the surface atomic structure shows the same symmetry properties as present in the bulk. The surface atomic rearrangement is then referred to as a relaxation. For semiconductors the surface often exhibits a lower symmetry, and the atomic rearrangement is denoted a reconstruction.

A clean, smooth, crystalline surface can experimentally be created in different ways, e.g. by epitaxial growth or by cleavage. A sharp low energy electron diffraction (LEED) pattern is an indication of a good overall crystalline quality. On a microscopic scale, however, a surface always exhibits various defects; such as steps, kinks, and point defects. On the atomic scale most of the atoms may still be found in an ordered two-dimensional lattice over areas of the order of the coherence length of the experiment (e.g. photoemission). It is therefore a good approximation to use a perfect crystal surface as a model for the interpretation of many experimental results.

There exists today a large variety of experimental techniques for investigating the atomic structure of surfaces. Some of these give a fairly direct picture of the real surface. To this category belong transmission electron microscopy (TEM), scanning tunnelling microscopy (STM) and atomic force microscopy (AFM). Other techniques give more indirect information and must be supported by numerical evaluation. Examples of these techniques are surface extended X-ray absorption fine structure (SEXAFS) and photoelectron diffraction (PhD). A third category of methods is the diffraction methods, which give information about the reciprocal lattice. The experiments are performed by means of X-rays, electrons, atoms or molecules.

Data for atomic positions have today been reported for many semiconductor surfaces. The agreement between various authors is good from a qualitative point of view, although the absolute positions usually differ [1].

\section{The electronic structure of surfaces}

In the middle of the 1960's Hohenberg, Kohn, and Sham [2-4] showed that the electronic ground state of a solid can be described exactly by a set of one-body, Hartree-like equations using a local, effective potential $V_{\text {eff }}(\boldsymbol{r})$ :

$$
\left[-\frac{\hbar^{2} \nabla^{2}}{2 m}+V_{\mathrm{eff}}(\boldsymbol{r})\right] \psi_{i}(\boldsymbol{r})=\varepsilon_{i} \psi(\boldsymbol{r})
$$

$V_{\text {eff }}(r)$ can be split into two contributions $V_{\text {eff }}(r)=V_{c}(r)+V_{\text {xc }}(r)$. Here $V_{c}(r)$ is the sum of the Coulomb potential from all the nuclei and the average Coulomb potential from all the electrons (Hartree contribution). $V_{\mathrm{xc}}(r)$ represents the exchange-correlation potential describing the effect of the correlated movements among the electrons. The formalism is referred to as the density functional theory (DFT). 
It states in particular that the total ground state energy of the sample can be expressed as a universal functional of the one-electron density only. The explicit form of the functional is not known and the common approximation to DFT is the local density approximation (LDA). Here one assumes that the density varies slowly and that it accordingly can be described by a local function. The LDA has been successfully applied to a variety of systems. It also works surprisingly well at surfaces where the density variation is not slow. The explanation is that the exchange-correlation energy is not sensitive to the detailed shape of the exchange-correlation hole but only to the spherical average of it. The important quantity is the amount of repelled charge that is one electron as required by DFT.

The DFT can strictly only be used to calculate the electron density, the total energy and other ground state properties. The eigenvalues of Eqs. (1) for the valence electrons in a crystal constitute the conventional band structure $\varepsilon_{i}(k)$, which usually is calculated within the LDA. It is important to realise that the individual eigenvalues $\varepsilon_{i}$ have no direct physical interpretation but are only Lagrangian parameters.

A very powerful method to test the electronic structure is photoelectron spectroscopy, PES. In this technique the sample is irradiated by monochromatic light and the photoemitted electrons are analysed with respect to their kinetic energies. The number of detected electrons per energy interval as a function of their kinetic energy is called an energy distribution curve, EDC. Structure in an EDC can be attributed to a binding energy $E_{\mathfrak{B}}$ by the use of the equation

$$
E_{\mathrm{B}}=h \nu-E_{\mathrm{kin}}-e \phi,
$$

where $h \nu$ is the photon energy and $e \phi$ is the work function. The relationship is illustrated in Fig. 1. The PES technique was discussed in some detail at the last School in this series [5]. We therefore here only stress the fact that an excited state, rather than the ground state, is probed. In many cases, in particular for metals which exhibit strong screening, the LDA band structure energies are good

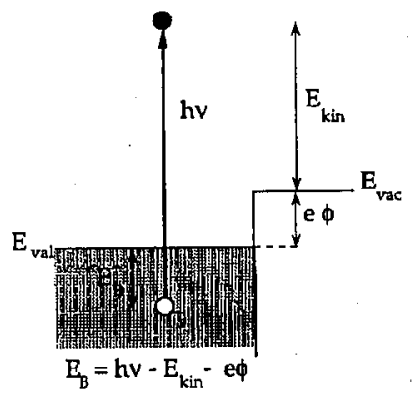

Fig. 1. Illustration of energy conservation in photoelectron spectroscopy on a semiconductor. $E_{\mathrm{B}}$ is the electron binding energy relative to the valence band maximum $E_{\mathrm{v}}, h \nu$ is the photon energy, $E_{\text {kin }}$ is the electron kinetic energy in vacuum, and $e \phi$ is the work function. 
approximations to excitation energies. For serniconductors, however, excitation effects give rise to small but observable deviations from the conventional band structure description. The error is in general not due to the approximations in the LDA but to the neglect of the modification of the exchange-correlation potential around the created electron and hole. Rigorously, excited states are derivable from

$$
\left[-\frac{\hbar^{2} \nabla^{2}}{2 m}+V_{\mathrm{c}}(r)\right] \phi_{i}(r)+\int \mathrm{d}^{3} r^{\prime} \Sigma\left(r, r^{\prime}, E_{i}\right) \phi_{i}\left(r^{\prime}\right)=E_{i} \phi_{i}(r)
$$

where $\Sigma\left(\boldsymbol{r}, \boldsymbol{r}^{\prime}, E_{i}\right)$ is the non-local, energy-dependent and non-Hermitian (complex) self-energy. These self-energy effects have been successfully incorporated [6] in band structure computations by the use of the so-called GW-approximation [7], but this involves quite an effort. The real part $\Sigma_{\mathrm{r}}(E, k)$ shifts the energy bands while the irnaginary part $\Sigma_{\mathrm{i}}(E, k)$ introduces the lifetirne broadening. A further approximation is to neglect the $k$-variation of the self-energy so that only the energy-dependence remains. The LDA band structure should then be uniformly "stretched" to higher energies above the Fermi level $E_{F}$ to reproduce the experimental results. Correspondingly, the holes will be observed at lower binding energies than predicted by the LDA. The latter effect is usually of the order of a few tenths of an $\mathrm{eV}$. The excitation effects become dramatic at the band gap: most of the observed band gap is attributed to final state effects in the experiment.

An alternative approach to $a b$ initio, seff-consistent methods is offered by (semi-)empirical techniques. These paraneterized methods are simple to use and often give a direct insight into the qualitative physical situation. We will now use such a formalism to describe the electronic and geometric structure at surfaces of tetrahedrally co-ordinated semiconductors (diannond and zinc-blende lattices). Linear combinations of atomic orbitals $\varphi_{i}$ (LCAO) are taken as basis functions and treated as an orthogonal set (tight-binding approximation, TBA) [8]. A "rninimal basis set" is used, namely the occupied $s$ - and p-orbitials (4 orbitals for diamond and 8 for zinc-blende structure). The Hamiltonian 3-centre rratrix elements $H_{i j}=\left\langle\varphi_{i}|H| \varphi_{j}\right\rangle$ are approximated by 2-centre integrals which are treated as parameters: $s s \sigma, s p \sigma, p p \sigma$, and $p p \pi$. Only interactions between nearest and next-nearest neighbours are included. The errors arising from all these approximations are fortunately to a large extent absorbed in the parameters so that an effective Hamiltonian still describes the experimental findings well.

For a qualitative discussion of the surface electronic structure it is instructive to use so-called $s p^{3}$ hybrid states $h_{i}, i=1,4$ instead of atomic orbitals. These functions are linear combinations of the atomic orbitals, $h_{i}=a_{1} s+a_{2} p_{x}+a_{3} p_{y}+$ $a_{4} p_{z},\left|a_{i}\right|=1$. The eigenvalues will of course be unchanged by this similarity transformation, but the hybrid wave functions display a more intuitive picture for the (surface) electronic structure. The new basis set, illustrated in Fig. 2, consists of orbitals forming directed lobes between the atoms. The largest overlap integral $\beta$ is between the two hybrids pointing straight towards each other. Taking only this interaction into account we arrive at the extreme approxirnation called the molecular model $(\mathrm{MM})$. The atomic hybrid energies $E_{\mathrm{h}}=\left(E_{s}+3 E_{p}\right) / 4$ (where $E_{s}$ and $E_{p}$ are atorric energies) are split into a bonding and an antibonding level with energies $E_{A, B}=E_{\mathrm{h}} \pm \beta$. Some of these bonds are broken at the surface, resulting in 


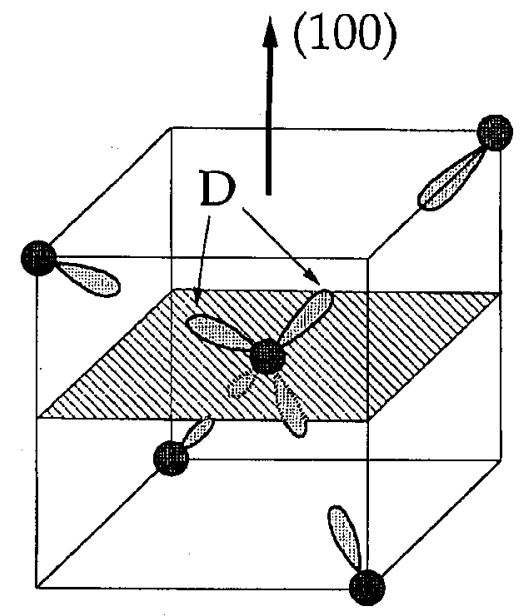

Fig. 2. Schematic picture of $s p^{3}$ hybrid states in a diamond type semiconductor. For a (100) surface two hybrids $D$, "dangling bonds" stick out of the surface.

unpaired lobes sticking out of the surface and forming so-called "dangling bonds". For the diamond (100) surface these bonds are denoted $D$ in Fig. 2. In the MM the bonds give rise to localised states, surface states, in the middle of the gap. When the other interactions between the hybrids are turned on the levels broaden and develop into the band structure of the material. The dangling bonds interact directly and through the solid, which gives rise to a $k$-dispersion parallel to the surface.

For relaxed atomic positions the atomic state composition of the hybrids is changed. As a result the surface state energy varies linearly with the displacement. In addition the elastic energy varies quadratically. We now arrange so that alternate hybrids have two electrons and are empty, respectively. This model [9] predicts that the doubly occupied hybrid moves out of the surface and becomes $s$-like, while the empty hybrid moves inwards and becomes $p$-like. These movements can be viewed as a type of Jahn-Teller distortions. The described relaxation mechanism is reproduced in full scale calculations and observed experimentally as illustrated below.

Two categories of valence and conduction electronic states appear in the surface region. Firstly, we have localised wave functions, so-called surface states or surface resonances. They decay away from the surface region and vanish a few (1-10) atomic layers inside the solid. The mentioned "dangling bonds" belong to this category. Secondly, a surface density of states $N_{\mathrm{S}}(E)$ is built up from the interference of the Bloch waves coming from the interior of the bulk and being reflected at the surface barrier. As the eigenenergies $\varepsilon_{n}(k)$ do not depend on $r$ they cannot change in the surface region. It is the local density, described by $\left|\psi_{k}(r)\right|^{2}$, which modulates the bulk density of states $N_{\mathrm{B}}(E)$ :

$$
N_{\mathrm{B}}(E)=\int \mathrm{d}^{3} k \delta\left(E-\varepsilon_{n}(k)\right),
$$




$$
N_{\mathrm{S}}(E)=\int \mathrm{d}^{3} k\left|\psi_{k}(r)\right|^{2} \delta\left(E-\varepsilon_{n}(k)\right) .
$$

The surface density of states have been very little studied so far. The focus has mainly been on surface states and resonances.

Band structure methods cannot be directly applied to the surface problem because the $k$-vector perpendicular to the surface is not defined. A direct approach is to use Green's function techniques. This choice is computationally very efficient compared to other alternatives. Furthermore, the method is conceptually very tractable and relevant results can easily extracted. The Green function matrix is defined from

$$
G(E)=\lim _{\eta \rightarrow 0}(E-H+\mathrm{i} \eta)^{-1}
$$

where $H$ is the Hamiltonian matrix. $G(E)$ for the semi-infinite system can be calculated from Dyson's equation $G=G_{0}+G_{0} U G$ [10], where $G_{0}$ refers to the bulk and $U$ to the surface. Technically the crystal is divided into identical layers parallel to the surface, each layer containing one or more atomic planes. The local density of states $\rho\left(E, k_{\|}, r\right)$ is obtained in any layer from

$$
\rho\left(E, k_{\|}, r\right)=-\frac{1}{\pi} \lim _{\eta \rightarrow 0} \operatorname{Tr} \operatorname{Im} G\left(E+\mathrm{i} \eta, k_{\|}, r\right) .
$$

By restricting the trace surnmation to a specific basis function the partial density of states is found.

In the sketched formalism the valence and conduction states are described by an effective band structure, quasi-particles. For the non-dispersive core excitations we abandon the quasi-particle picture and consider the conservation of the energy of the total system. Due to relaxation effects around the localised core hole the measured binding energy $E_{\mathrm{B}}$ may be several $\mathrm{eV}$ smaller than predicted by the ground state. We write

$$
E_{\mathrm{B}}=E_{\mathrm{f}}^{\text {tot }}-E_{\mathrm{i}}^{\text {tot }}=\varepsilon_{n}-E_{\text {relax }}
$$

where $\varepsilon_{n}$ are one-electron energies. We note that the measured binding energy $E_{\mathrm{B}}$ is dependent on both the initial and final state total energies, $E_{\mathrm{i}}^{\text {tot }}$ and $E_{\mathrm{f}}^{\text {tot }}$ respectively. Note also that the concept of "final state" was differently used above in the quasi-particle description of valence bands. There it referred to an electron state as described by the unoccupied (effective) band structure. For core hole excitation the final state refers to the total final state where both the core hole as well as the valence electrons play explicit roles.

The core electrons are affected by the presence of the surface. Small negative or positive energy shifts relative to the bulk value are observed. As will be further discussed below there are both initial contributions to this effect in terms of charge transfer as well as final state effects coming from the locally modified screening of the hole.

\section{Example 1: core levels on $\mathrm{Si}(100)$}

The ideal $\operatorname{Si}(100)(1 \times 1)$ surface is shown in Fig. 3. The surface atoms are next nearest neighbours and have two dangling bonds each, both half occupied. The real surface shows a variety of reconstructions, denoted by $2 \times 1$, 
$(0)$

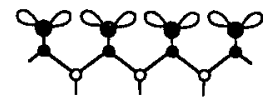

(b)

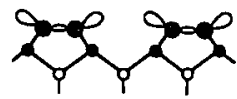

(c)

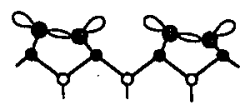

(d)

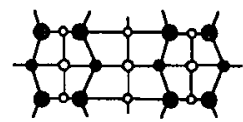

Ideal

$\underset{\text { dimer }}{\text { Symmetric }}$

Asymmetric
dimer

Asymmetric

dimer

top view

Fig. 3. Dimer formation at the (100) surface of a diamond type semiconductor. Si(100) and $\mathrm{Ge}(100)$ : As reconstruct according to part (c) and (b) respectively.

$c(4 \times 2), p(2 \times 2) \ldots$ relative to the $1 \times 1$ unreconstructed cell. It is now generally agreed [11-13] that these reconstructions result from different arrangements of the so-called asymmetric dirners which was originally proposed by Chadi [14]. The $(2 \times 1)$ reconstructions case is also illustrated in Fig. 2. The model described in Sec. 3 above is qualitatively supported by observations of two surface bands; one occupied band arising from dangling bonds on the up atoms and one unoccupied band arising from dangling bonds on the down atoms.

The $2 p$ core levels on $\mathrm{Si}(100)$ have been studied by several authors. We choose here to reproduce the recent photoemission results by Landemark et al. [15]. The observed spectra were decomposed by a least squares fitting procedure using spin-orbit split Voigt functions. Except for the bulk contribution four surface shifted peaks were observed. For clarity we show in Fig. 4 the decomposition of only one spin-orbit component. Furthermore only two surface components are shown, namely those related to the up and down surface atom respectively. The other two components were associated with subsurface atoms. We know that in the ground state the up atorn is negatively charged, while the down atom is positively charged. The surrounding atoms also exhibit charging, but that contribution (Madelung energy) does not overcompensate the intra-atomic part. We thus expect to a first approximation a decrease (an increase) in the $2 p$ binding energy for the up (down) atom. This is also observed, although the shift for the down atom is quite small.

For a more accurate description the effect of the detailed hole screening at the surface must be included. In a sirnple model one would think that in general the screening, or rather the polarization, at the surface is reduced because there is no contribution from the vacuum. However, this is not necessarily true. When a core hole is created on the down atom, the unoccupied dangling bond on this atom is pulled down and becomes filled. This process allows an efficient screening and decreases the binding energy. Obviously the same process does not occur on the up atom because the dangling bond is already occupied in the ground state. Pehlke and Scheffler [16] computed the effect of final state screening in the static limit using a DFT-LDA approach. As seen in Fig. 4 the effect is considerable. In 


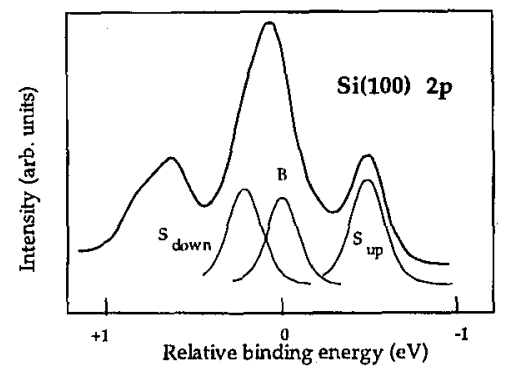

a)

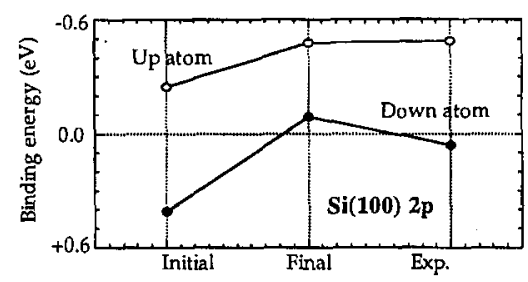

b)

Fig. 4. (a) The $2 p$ core levels as measured and analysed by Landemark et al. [15]. For clarity only some contributions are shown here. (b) The binding energy of the $2 p$ states on the up and down atoms of the dimer as calculated by Pellke and Schäfler [16].

particular the down-atorn peak is shifted due to screening with about $0.5 \mathrm{eV}$, so that it appears close to the bulk peak. We thus find that the exact value of the surface core levels is a complicated function of the strongly varying local screening. The qualitative shift is however usually given by the charge transfer in the ground state.

\section{Example 2: valence states on $\operatorname{InP}(110)$}

As discussed in Sec. 3 surface states may appear in the valence and conduction band region. Traditionally these states are termed surface resonances when they are degenerate with bulk states. The "resonances" differ from the "states" essentially only through their increased lifetime broadening.

We have applied the Green function method described in Sec. 2 to $\operatorname{InP}(110)$ within the parameterized LCAO model [17]. Including next-nearest neighbour interactions nine parameters are required. These were chosen such that the bulk band structure, as observed in angle-resolved photoemission, was reproduced [18]. Thus the excitation effects are effectively included. The (110) surface of zinc-blende semiconductors is found to relax so that the outermost anion moves outwards and the outermost cation moves inwards [1]. Although there are some scatters in the experimental data the anion-cation angle seerns well-established to about $28^{\circ}$.

To limit my discussion here we analyse the results in the $\bar{M} \bar{X}$ direction only. The data for the other zone boundaries were equally successful. Peak positions in the spectral functions for the outermost layers have been plotted in Fig. 5. Five bands are found. The shadowed region is the surface projected bulk density 

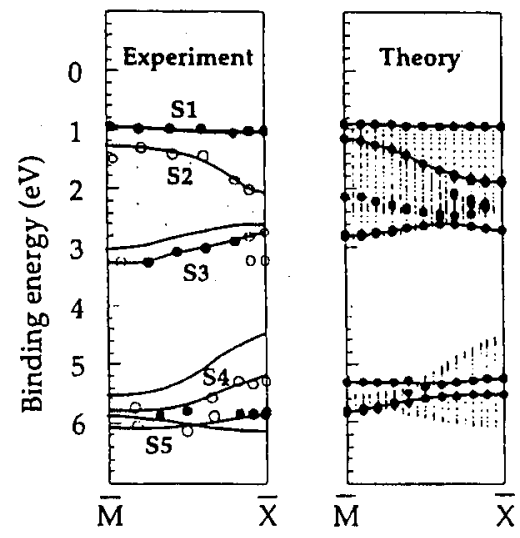

Fig. 5. The procedure to derive $k_{\|}$of a surface excitation.

of states. The calculations are compared in the same figure with results obtained from angle-resolved photoemission in Fig. 5. The (110) surface was prepared by cleavage in situ. The spectra were recorded with an angular and energy resolution of $2^{\circ}$ and $0.3 \mathrm{eV}$ respectively.

Excitations from the bulk was singled out by the help of bulk band structure data obtained within the LCAO model. The 2-dimensional band structure $E\left(\boldsymbol{k}_{\|}\right)$ of the surface states (resonances) can be directly obtained from the experimental data through the energy conservation relation (2) together with conservation of crystal momentum parallel to the surface

$$
\left|k_{\|}\right|=\sqrt{2 m E_{\text {kin }}} \sin \theta / \hbar \text {. }
$$

Here $n$ is the free electron mass of the electron and $\theta$ - the emission angle according to Fig. 6.

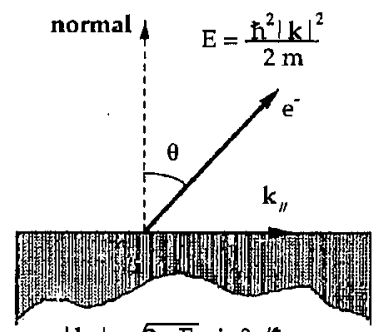

$\left|k_{\mu l}\right|=\sqrt{2 \mathrm{mE}} \sin \theta / \hbar$

Fig. 6. Surface related features as calculated [17] and measured [18] in the $\bar{M} \bar{X}$ azimuth of the $\operatorname{In} P(110)$ surface.

We observe in Fig. 5 that the agreement between theory and experiment is very good. From the local and partial density of states the character of the surface states can be deduced. Therefore for instance the $S_{1}$ state is associated with the dangling bond hybrid of $p$-character located on the $\mathrm{P}$ atom as discussed 
with the dangling bond hybrid of $p$-character located on the $\mathrm{P}$ atom as discussed above. The $S_{4}$ state have about equal In $s$ and $\mathrm{P} p$ character and could only be found for a reduced coupling (compared to the bulk) between the first and second atomic layers. The experiments were performed and analysed [18] before the theory was worked out. Therefore a further analysis of the experimental data may reveal the calculated structure in the region around $-2.5 \mathrm{eV}$. In fact due to poor interaction between the experimental and calculation processes very few surface band structures have yet been established to a larger degree in literature.

\section{Example 3: core levels and valence states on Ge(100):As}

The physics at the interface between two semiconductors (heterojunction) plays an important role for the understanding of the performance of semiconductor devices. The electrical and structural properties of a heterojunction are very much determined already at the formation of the first monolayer in the growth process. We can therefore learn a lot by studying monolayers by photoelectron spectroscopy. As an example we relate here an investigation of the chemisorption of As on $\mathrm{Ge}(100)[19]$ as part of a study of the $\mathrm{Ge} / \mathrm{GaAs}$ interface.

The clean $\mathrm{Ge}(100)$ surface is characterised by asymmetric dimers in the same way as described for $\mathrm{Si}(100)$ above. As was deposited from an effusion cell in an MBE system onto the Ge substrate held at $450^{\circ}$. The LEED pattern showed a two-domain $2 \times 1$ structure. The As $3 d$ core level consisted of one component only while the Ge $3 d$ level revealed two components. These findings suggest the existence of a single monolayer of As in the form of AsGe. Angle-resolved spectra from
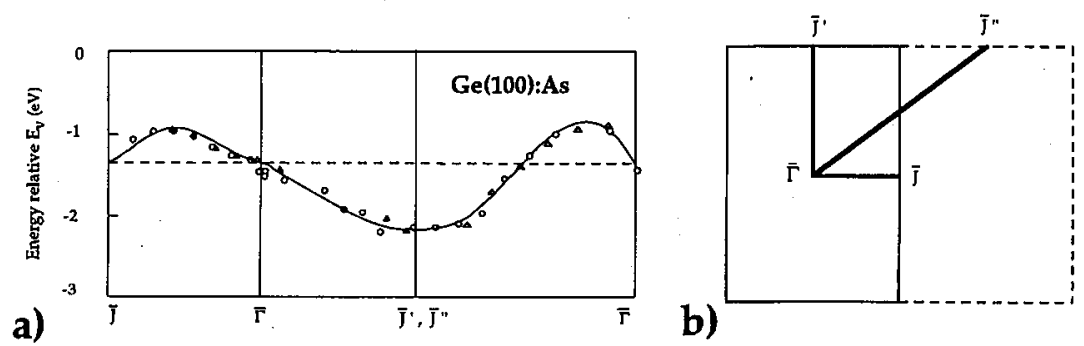

Fig. 7. (a) The surface Brillouin zone of the $2 \times 1$ reconstructed $\mathrm{Ge}(100)$ : As surface. (b) The dangling bond dispersion as deduced by Morar et al. [19].

the valence band were recorded in two azimuths. From these data the dispersion relation for the As dangling bond could be extracted, see Fig. 7. The results are in agreement with a model where the As atoms form symmetric dimers, see Fig. 3. The interaction between the dangling bonds occurs mainly via the substrate. There should be a splitting into bonding and antibonding states, but it is obviously too small to be observed. At lower energies bands are found (not shown here), which are attributed to the symmetric and antisymmetric combinations of the As dimer bond. The findings for the $\mathrm{Ge}(100) /$ As system are analogous to what has been found for $\mathrm{Si}(100) /$ As [20]. 


\section{Conclusions}

Photoelectron spectroscopy provides a very powerful technique for studies of the electronic structure of surfaces and overlayers. Also interfaces can be investigated provided they do not occur too far below the surface, i.e. less than a few atomic layers. Still however there has not so far been reported a "full electronic characterization" of any semiconductor surface. The experimental results are usually restricted in energy and $k$-space. Moreover, there is typically only a partial agreement between different experimental results, between different theoretical results and between theoretical and experimental results. The experimental and theoretical tools are today advanced and reliable. What is needed is a coordinated experimental and theoretical effort. The results presented here on $\operatorname{InP}(110)$ is an example of this.

The use of core electron spectroscopy is a valuable complement, in particular for indirect geometrical structural information. The method relies on decomposition techniques and rnoreover provides only a few numbers as the final result. Considering correctly the contributions of charge transfer and final state relaxation to the measured binding energies, core level spectroscopy can be turned into an even more valuable tool for establishing the electronic structure of semiconductor surfaces.

\section{References}

[1] F. Bechstedt, R. Enderlein, in: Semiconductor Surfaces and Interfaces, Akademie-Verlag, Berlin 1988, p. 270.

[2] P. Hohenberg, W. Kohn, Phys. Rev. B 136, 864 (1964).

[3] W. Kohn, L.J. Sham, Phys. Rev. A 140, 1133 (1965).

[4] L.J. Sham, W. Kohı, Phys. Rev. 145, 561 (1966).

[5] P.O. Nilsson, Acta Phys. Pol. A 82, 201 (1992).

[6] M.S. Hybertsen, S.G. Louie, Phys. Rev. Lett. 55, 1418 (1985); R.W. Godby, M. Schlüter, L.J. Sham, Phys. Rev. B 37, 10159 (1988).

[7] L. Hedin, S. Lundqvist, Solid State Phys. 23, 1 (1969).

[8] W.A. Harrison, in: Electronic Structure and the Properties of Solids, W.H. Freeman and Company, San Francisco 1980, p. 77.

[9] See Ref. [8], p. 233.

[10] See e.g. M.P. Lopez Sancho, J.M. Lopez Sancho, J. Rubio, J. Phys. F, Met. Phys. 15, 851 (1985).

[11] N. Roberts, R.J. Needs, Surf. Sci. 236, 112 (1990).

[12] J. Dabrowski, M. Scheffler, Appl. Surf. Sci. 56-58, 15 (1992).

[13] R.A. Wolkow, Phys. Rev. Lett. 68, 2636 (1992).

[14] D.J. Chadi, Phys. Rev. Lett. 43, 43 (1979).

[15] E. Landemark, C.J. Karlsson, Y.-C. Chao, R.I.G. Uhrberg, Phys. Rev. Lett. 69, 1588 (1992).

[16] E. Pehlke, M. Scheffler, Phys. Rev. Lett. 71, 2338 (1993).

[17] Y. Khazmi, P.O. Nilsson, J. Kanski, to be published. 
[18] H. Qu, J. Kanski, P.O. Nilsson, U.O. Karlsson, Surf. Sci. 255, 237 (1991).

[19] J.F. Morar, U.O. Karlsson, R.I.G. Uhrberg, J. Kanski, P.O. Nilsson, H. Qu, Appl. Surf. Sci. 41/42, 312 (1989).

[20] R.I.G. Uhrberg, R..D. Bringans, R.Z. Bachrach, J.E. Northrup, Phys. Rev. Lett. 56, $520(1986)$. 